\title{
Functional and Anatomical Imaging for Early Diagnosis of Tumor Induced Osteomalacia
}

\author{
Ghazala Malik ${ }^{1}$ and Fatima Sultan ${ }^{2}$ \\ ${ }^{1}$ Consultant Radiologist, United Lincolnshire Hospitals NHS Trust, United Kingdom \\ ${ }^{2}$ Shalamar Medical College, Shalamar Institute of Health Sciences, Pakistan
}

Submission: March 30, 2017; Published: April 10, 2017

"Correspondence Address: Ghazala Malik, Consultant Radiologist, United Lincolnshire Hospitals NHS Trust 101 Manthorpe Road, Grantham NG31 8DG, United Kingdom, Email: ghazalamalik97@gmail.com

\begin{abstract}
Oncogenic osteomalacia, or tumor-induced osteomalacia (TIO), is an acquired paraneoplastic syndrome being associated with benign or in rare cases malignant mesenchymal. Patients with TIO often present with many years of nonspecific but progressive symptoms commonly with bone pain, muscle weakness, reduced height, and multiple fractures. Due to this nonspecificity, patients are often misdiagnosed with a variety of musculoskeletal, rheumatologic diseases and sometimes even having psychiatric disorders. Not only that these tumors are rare but also finding the tumor can be a major diagnostic challenge and may need total body magnetic resonance imaging, computed tomography and scintigraphy using radio labelled somatostatin analogue and sometimes even venous sampling. Majority of clinicians, radiologists and pathologists are not aware of this rare disease because mostly the cases are described in rheumatology practice. We provide mini review of the current literature by having looked at the different studies done including case reports to conclude the best practice for the diagnosis and treatment of this rare entity.
\end{abstract}

Keywords: Magnetic Resonance Imaging; Positron Emission Tomography; Computed Tomography; Venous Sampling

\section{Introduction}

Tumors that can lead to this syndrome are classified on the basis of histology as phosphaturic mesenchymal tumors mostly mixed connective tissue PMTMCT, but also other morphology like osteoblastoma like tumors, ossifying fibrous like tumors, or nonossifying fibrous like tumors .Weidner N. Reviewed 16 tumours to establish four different morphologic patterns with further subgroups mostly the first group with ten sub types [1]. Decades after the discovery of fibroblast growth factor mesenchymal tumor were found to be related to fibroblast growth factor 23 (FGF23), which is responsible for hypophosphataemia and phosphaturia occurring in this paraneoplastic syndrome [2]. Hypophosphataemia, phosphaturia and elevated alkaline phosphatase are the important laboratory readings that may guide to more precise investigations and better diagnosis $[3,4]$. Regardless of tumour morphology, the hallmark of the diagnosis which forms the basis of all diagnostic modalities is the association of the tumor with the clinical syndrome of TIO, which includes an elevation in plasma FGF23 and its disappearance after tumor resection. As tumours are typically very small in size and slow-growing, detecting and locating these tumors is quite challenging [5].
Clunie GP et al. studied four cases confirming that slow recognition of acquired hypophosphataemic osteomalacia and TIO resulting in unnecessary and prolonged morbidity. These cases emphasize that osteomalacia may precede the discovery of a tumor and illustrate that the site and nature of tumors and their complete resection is often difficult to asses [6,7]. As a result, the time from osteomalacia to identifying the associated tumour averages a period of 5 years and in some studies 7 years. An added problem is that tumor behavior varies from case to case. Seijas R et al. reported two cases with surprisingly different outcomes one showing complete cure while other resulted in incomplete resection and later on recurrence with multiplicity [8].

\section{Discussion}

TIO was first described in 1947 by Robert McCance, who treated a patient having low phosphorous levels, bone pain, with high doses of vitamin D but the symptoms did not completely resolve until a tumor in femur bone was removed. In 1959, Andrea Prader treated rickets in an 11-year-old girl who had decreased tubular phosphate reabsorption however normal kidney function. A tumour, giant cell granuloma, was diagnosed 


\section{Cancer Therapy \& Oncology International Journal}

in a rib and removed resulting in healing of rickets. More than 300 cases of TIO have been reported in the literature $[9,10]$.

The first evidence of a circulating factor that could cause phosphate wasting in phosphaturic disorders was set by Meyer et al. and Nesbitt et al. in mice and by Miyauchi et al. who induced hypophosphatemia in mice by transplanting a tumour removed from a patient $[2,11]$. FGF23 binds to target proximal tubule cells via FGF receptor and inhibits renal phosphate reabsorption FGF23 is also a regulatory hormone for 1.25-vitamin D and leads to a decreased concentration of the vitamin in blood under physiological conditions, FGF23 is secreted predominantly by bone and undergoes degradation by proteolytic enzymes. By contrast, tumours secrete FGF23 several hundred-fold higher than normal levels, leading to dysregulation of FGF23 degradation pathway $[12,13]$.

PMT tumours usually occur in bones or soft tissues. The most common diagnosis for these tumours has been hemangiopericytoma [14], but it has also included hemangioma, sarcomas, ossifying fibromas, granulomas, giant cell tumours and osteoblastomas. Most of them are believed to be benign, although histopathologically malignant have been also described and also while typically benign, malignant presentation and metastases can occur. While metastases are rare, infiltration of surrounding connective tissue is typically present, which has significant implications for surgical management and recurrence. It also emphasises importance for wide surgical margins to avoid persistence or reoccurrence $[3,7]$. Uramoto $N$. et al, reported a case of a recurrent malignant PMT variant which arose in the tongue. In accordance with the tumor recurrence and resection, the hypophosphatemia progressed and didn't improve. However, hypophosphatemia did not progress after receiving radiation therapy at the second recurrence even though the recurrent tumor gradually increased its size. These results suggest clinical feature of malignant PMT could be changed by radiation therapy. Thus, this report could add an insight to the nature of PMT $[13,15]$.

Quoting Jiang et al. review showed, tumors originate in bones (40\%) and soft tissues (55 \%) most common limbs and craniofasial [16] and less common locations are the vertebra, knee, hand, chest, abdomen, groin, perineum and gluteal region even in organs such as the liver, tongue, thyroid and lungs. Folpe $\mathrm{AL}$, described osteomalacia-associated mesenchymal tumors of 32 cases and describing craniofascial origion [17]. Quoting Jiang et al. who reviewed 308 tumour-induced osteomalacia cases reported in English literature between 1987 and 2011, about $46 \%$ of reported cases of TIO have occurred in females and $56 \%$ in males, with a mean age of 45 years when definitive diagnosis was made .Patients with TIO often present with bone pain, muscle weakness, reduced height, and multiple fractures, primarily in the ribs, vertebral bodies, and femoral neck. Bumard $\mathrm{H}$ and co workers presented a case with bilateral insufficiency fractures of the proximal femur often have a pathological basis of hemangiopericytoma. Diagnosis of rare causes of insufficiency fractures can be challenging when the causative factor is a tumour [18].

A systematic approach to hypophosphatemia helps in the early recognition and treatment of TIO. Impaired renal phosphate reabsorption is the biochemical hallmark of the disease with normal or low levels of 1,25-dihydroxyvitamin D, elevated levels of alkaline phosphatase and normal circulating levels of calcium and mostly normal or high parathormone $[5,6]$. When testing antigen expression, FGF23 is positive in about $70 \%$ of all the cases studied, and the proliferating cells within the tumour are usually the source of FGF23 [6]. Somatostatin receptors have also been found to be present in many TIO tumors [19]. Localization of FGF23-producing tumors can be challenging and a step-wise approach, including functional imaging, followed by anatomical imaging, and if necessary selective venous sampling may significantly improve success in tumor localization [6].

Establishing the diagnosis of an FGF23-producing phosphaturic mesenchymal tumor requires an adequate integration of the clinical picture in correlation with radiology and histology in a multidisciplinary setting, since radiology and histology show considerable overlap with other entities that need different treatment and harbor other clinical outcomes. A stepwise approach is advocated, first performing functional tests that include scintigraphy using radiolabelled somatostatin analogue (such as 99mTc-Tektrotyd). Tumors associated with osteomalacia variably express five somatostatin receptors (SSTR1-5), allowing SSTR-based functional imaging by somatostatin analogue scintigraphy or positron emission tomography [19-21].

Octreotide is somatostatin analogue that can be radiolabelled in an attempt to detect tumours that express somatostatin receptors [20]. Octreotide scanning is commonly performed with 111In-labelled pentatreotide. Octreotide scintigraphy is successfully used to locate tumors in up to $95 \%$ of patients with TIO.A study in china by Yang et al, diagnosing and managing 39 cases of TIO, suggesting that the syndrome is an important cause of adult-onset hypophosphatemic osteomalacia and ${ }^{99} \mathrm{Tc}^{\mathrm{m}}$-OCT scintigraphy is an effective method to detect the tumors that cause TIO [22]. Jan de Beur SM and co workers did Indium-111 labeled pentetreotide imaging in seven patients with oncogenic osteomalacia. In five patients, it was identified a mesenchymal tumour, and clinical improvement occurred after tumour resection suggesting that 111 In-pentetreotide imaging effectively detects occult mesenchymal tumours and facilitates surgical treatment of oncogenic osteomalacia [23].

Despite this success, there are several limitations of this imaging technology. Inflammatory reactions or a fracture will be associated with a false-positive scan. Somatostatin analogues scintigraphy is also limited by planar two-dimensional imaging and relatively poor spatial resolution, which is particularly 


\section{Cancer Therapy \& Oncology International Journal}

problematic given that TIO tumours are often very small. Singlephoton emission tomography (SPECT) or hybrid SPECT/CT enables three-dimensional imaging and better tumour contrast but is time-consuming and therefore limited to areas of suspected abnormality rather than a whole body survey. Somatostatin analogue positron emission tomography can dramatically improve the spatial resolution and lesion detectability. 68Gaoctreotide PET/CT may have an important role in localization of the culprit lesion, and may potentially reduce significant delay in establishing the diagnosis of TIO [24,25].

FDG-PET/CT can confirm a lesion or identify a lesion that was not initially seen on octreotide scintigraphy. FDG-PET/CT has been proven to be sensitive but not a specific method of identifying FGF23-producing tumors. PET/CT using 68-gallium is expected to be much more specific and informative for this kind of tumors but is often not available in many centers. Agarwal et al. compared the utility of F-FDG PET/CT and Ga DOTATATE PET/CT to detect the site of primary tumor in patients with suspicion of TIO in 6 patients in which former could detect 3 cases while latter could detect 5 cases [24]. In another study on 54 patients Zhang J and co workers observed (68) Ga DOTATATE PET/CT scans were positive in 44 patients, among which, 33 had surgery to remove the lesions and their symptoms diminished promptly, and the serum phosphate levels became normal, which confirmed the diagnoses of TIO [22,25].

There are certain circumstances in which more certainty and testing are indicated. When multiple suspicious lesions are identified on functional imaging, particularly FGD-PET, each with a reasonable degree of suspicion or the suspicious lesion is located in an area where the indicated operation is associated with a high level of potential morbidity. In these cases, additional certainty and testing are indicated. Of particular utility is selective venous sampling with measurement of FGF23 [5].

Venous sampling has been attempted in localizing tumors in the absence of any suspicious lesions identified on either functional or anatomical imaging [26]. In a trial to test to localize tumors by venous sampling without a 'target' lesion which was suggested by anatomical or functional imaging, it was concluded to be not a useful approach [26]. Van Boekel etal. (2008) advocated a two step approach to venous sampling. They suggested that if a suspected tumor cannot be localized by imaging, whole body venous sampling can be performed with assessment of the average values for samples from different anatomical regions. If the average values in samples from a region appear to be higher, more detailed sampling is performed in the smaller branches of the veins in that region. In one patient studied by this approach, it appeared to suggest a particular region. However, in retrospect, the tumor was evident on an MRI that had been performed prior to venous sampling, so the utility of this approach is not clear. An additional approach that can be used for confirmation that a suspicious lesion identified on functional or anatomical imaging is the culprit tumor is aspiration of the lesion. Elevated FGF23 in the aspirate is diagnostic of a causative lesion. Inspection of cellular morphology consistent with that of a phosphaturic mesenchymal tumor, further supporting that the aspirated lesion represents the culprit lesion Sciubba et al. (2009)

Anatomic imaging (radiography, CT and MRI) should be performed to confirm the location of the tumor after suspicious lesions had been identified by functional imaging]. CT indicates deformations in skeletal bones, rib, bons, thoracic vertebrae and pelvic bone due to osteomalacia or stress fractures, compressive with pathologic kyphosis, stress fractures in both femoral heads and osteosclerotic tumour .Total body magnetic resonance imaging (MRI) is the accurate method to confirm the location of the tumour. It is important to scan the whole body, including extremities [4] as tumours can occur anywhere in the body. Despite all of the advances in imaging that are available today, tumour localization may not be successful. If this is the case, imaging studies should be repeated every 1-2 years.

\section{Treatment}

The treatment of choice for TIO is resection of a tumour with a wide margin, as recurrences of these tumours have been reported $[13,26]$. Tumour removal is always curative [24], and the recovery and improvement of the patients is relatively quick, FGF23 disappears rapidly from the circulation, and serum phosphate returns to normal by day 5 post operation. The prognosis depends on detecting the tumour and possibility to remove it widely. Nevertheless, the follow-up should be continued because the delayed metastasis can occur as it was described in few cases.

\section{Conclusion}

The diagnosis of TIO is a challenge and is commonly delayed for years due to the nonspecific nature of the presenting symptoms, failure to include serum phosphorus levels in blood chemistry and difficulty in identifying the responsible tumour. The professional evaluation of whole body images, including single-photon emission tomography enables identification and better tumour contrast which can be found in skeletal structures of whatever region of the human body. FGF23 may be of help to understand the underlying mechanisms of TIO and a measurement to follow up the disease course.

\section{Reference}

1. Weidner N (1991) Review and update: oncogenic osteomalacia-rickets. Ultrastruct Pathol. 15(4-5): 317-333.

2. Miyauchi A, Fukase M, Tsutsumi M, Fujita T (1988) Hemangiopericytoma-induced osteomalacia: tumor transplantation in nude mice causes hypophosphatemia and tumor extracts inhibit renal 25-hydroxyvitamin D 1-hydroxylase activity. J Clin Endocrinol Metab 67(1): 46-53.

3. Ogose A, Hotta T, Emura I, Hatano H, Inoue Y, et al. (2001) Recurrent malignant variant of phosphaturic mesenchymal tumor with oncogenic osteomalacia. Skeletal Radiol 30(2): 99-103. 


\section{Cancer Therapy \& Oncology International Journal}

4. Avila NA, Skarulis M, Rubino DM, Doppman JL (1996) Oncogenic osteomalacia: lesion detection by MR skeletal survey. AJR Am J Roentgenol 167(2): 343-345.

5. Chong WH, Molinolo AA, Chen CC, Collins MT (2011) Tumor-induced osteomalacia. Endocr Relat Cancer 18(3): R53-R77.

6. Clunie GP, Fox PE, Stamp TC (2000) Four cases of acquired hypophosphataemic ('oncogenic') osteomalacia. Problems of diagnosis, treatment and long-term management. Rheumatol Oxf Engl 39(12): 1415-1421

7. Sun Z, Jin J, Qiu G, Gao P, Liu Y (2015) Surgical treatment of tumorinduced osteomalacia: a retrospective review of 40 cases with extremity tumors. BMC Musculoskelet Disord 2015: 16:43.

8. Seijas R, Ares O, Sierra J, Perez-Dominguez M (2009) Oncogenic osteomalacia: two case reports with surprisingly different outcomes. Arch Orthop Trauma Surg. 129(4): 533-539.

9. Prader A, Illig R, Uehlinger E, Stalder G (1959) Rickets following bone tumor. Helv Paediatr Acta 14: 554-565.

10. Carpenter TO (2012) The expanding family of hypophosphatemic syndromes. J Bone Miner Metab 30(1): 1-9.

11. Meyer RA, Meyer MH, Gray RW (1989) Parabiosis suggests a humoral factor is involved in X-linked hypophosphatemia in mice. J Bone Miner Res Off J Am Soc Bone Miner Res 4(4): 493-500.

12. Ramon I, Kleynen P, Body JJ, Karmali R (2010) Fibroblast growth factor 23 and its role in phosphate homeostasis. Eur J Endocrinol Eur Fed Endocr Soc 162(1): 1-10.

13. Ledford CK, Zelenski NA, Cardona DM, Brigman BE, Eward WC (2013) The phosphaturic mesenchymal tumor: why is definitive diagnosis and curative surgery often delayed? Clin Orthop. 471(11): 3618-3625.

14. Fuentealba C, Pinto D, Ballesteros F, Pacheco D, Boettiger O, et al. (2003) Oncogenic hypophosphatemic osteomalacia associated with a nasal hemangiopericytoma. J Clin Rheumatol Pract Rep Rheum Musculoskelet Dis 9(6): 373-379.

15. Uramoto N, Furukawa M, Yoshizaki T (2009) Malignant phosphaturic mesenchymal tumor, mixed connective tissue variant of the tongue. Auris Nasus Larynx. 36(1): 104-105.
16. Savage CR, Zimmer LA (2009) Oncogenic osteomalacia from pterygopalatine fossa mass. J Laryngol Otol 123(9): 1052-10544

17. Folpe AL, Fanburg Smith JC, Billings SD, Bisceglia M, Bertoni F, et al. (2004) Most osteomalacia-associated mesenchymal tumors are a single histopathologic entity: an analysis of 32 cases and a comprehensive review of the literature. Am J Surg Pathol 28(1): 1-30.

18. Burnand H, Samuels A, Hagan I, Sawant N, Mutimer J (2012) Hip Bilateral subtrochanteric fractures in tumour-induced osteomalacia caused by a nasal haemangiopericytoma. Journal of clinical and experimental research on hip pathology and therapy 22(2): 227-229.

19. Houang M, Clarkson A, Sioson L, Elston MS, Clifton-Bligh RJ, et al (2013) Phosphaturic mesenchymal tumors show positive staining for somatostatin receptor 2A (SSTR2A). Hum Pathol 44(12) :2711-2718.

20. Duet M, Kerkeni S, Sfar R, Bazille C, Liote F, et al. (2008) Clinical impact of somatostatin receptor scintigraphy in the management of tumorinduced osteomalacia. Clin Nucl Med. 33(11): 752-756.

21. Moran M, Paul A (2002) Octreotide scanning in the detection of a mesenchymal tumour in the pubic symphysis causing hypophosphataemic osteomalacia. Int Orthop 26(1): 61-62.

22. Yan Jiang, Wei-bo Xia, Xiao-ping Xing, Barbara C Silva, Mei Li, et al (2012) Tumor-induced osteomalacia: An important cause of adultonset hypophosphatemic osteomalacia in China: Report of 39 cases and review of the literature. J Bone Miner Res 27(9): 1967-1975.

23. Jan de Beur SM, Streeten EA, Civelek AC, McCarthy EF, Uribe L, et al. (2002) Localisation of mesenchymal tumours by somatostatin receptor imaging. 359(9308): 761-763.

24. Agrawal K, Bhadada S, Mittal BR, Shukla J, Sood A, et al. (2015) Comparison of 18F-FDG and 68Ga DOTATATE PET/CT in localization of tumor causing oncogenic osteomalacia. Clin Nucl Med 40(1): e6-e10.

25. Zhang J, Zhu Z, Zhong D, Dang Y, Xing H, et al. (2015) 68Ga DOTATATE $\mathrm{PET} / \mathrm{CT}$ is an Accurate Imaging Modality in the Detection of Culprit Tumors Causing Osteomalacia. 40(8): 642-646.

26. Andreopoulou P, Millo C, Reynolds J, Kelly M, Brillante B, et al (2010) Multimodality Diagnosis and Treatment of Tumor Induced Osteomalacia. Endocrine Reviews 31(1): OR08-6S49.

\section{Your next submission with Juniper Publishers} will reach you the below assets

- Quality Editorial service

- Swift Peer Review

- Reprints availability

- E-prints Service

- Manuscript Podcast for convenient understanding

- Global attainment for your research

- Manuscript accessibility in different formats

( Pdf, E-pub, Full Text, Audio)

- Unceasing customer service

Track the below URL for one-step submission https://juniperpublishers.com/online-submission.php 